Recherches en didactique des langues et des cultures

Les cahiers de l'Acedle

6-1 | 2009

L'Alsace au cœur du plurilinguisme (2)

\title{
La conscientisation comme moyen \\ d'intériorisation de la catégorie de la détermination par les apprenants russophones $\mathrm{du}$ FLE
}

\section{Olga Spiridonova}

\author{
(2) OpenEdition \\ Journals \\ Édition électronique \\ URL : http://journals.openedition.org/rdlc/2206 \\ DOI : $10.4000 /$ rdlc. 2206 \\ ISSN : 1958-5772 \\ Éditeur \\ ACEDLE
}

Référence électronique

Olga Spiridonova, « La conscientisation comme moyen d'intériorisation de la catégorie de la détermination par les apprenants russophones du FLE ", Recherches en didactique des langues et des cultures [En ligne], 6-1 | 2009, mis en ligne le 01 avril 2009, consulté le 25 avril 2019. URL : http:// journals.openedition.org/rdlc/2206; DOI : 10.4000/rdlc.2206

Ce document a été généré automatiquement le 25 avril 2019

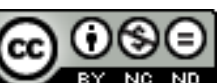

Recherches en didactique des langues et des cultures is licensed under a Creative Commons AttributionNonCommercial-NoDerivatives 4.0 International License 


\title{
La conscientisation comme moyen d'intériorisation de la catégorie de la détermination par les apprenants russophones du FLE
}

\author{
Olga Spiridonova
}

\section{Introduction}

1 Notre recherche s'inscrit dans le cadre des études sur l'enseignement / apprentissage de phénomènes grammaticaux d'une langue étrangère en milieu guidé. Plus précisément, il s'agit de l'apprentissage de la catégorie de la détermination par les apprenants russophones jeunes adultes faisant des études de cinq ans à l'université linguistique de Nijni Novgorod afin d'obtenir des diplômes d'interprètes de français et d'anglais.

2 Dans le présent article, nous expliciterons tout d'abord le choix de la conscientisation grammaticale pour l'apprentissage de l'article par les futurs interprètes. Ensuite, nous exposerons les hypothèses de la recherche. Après, nous parlerons de la méthodologie de la recherche qui comprendra la présentation du public et la description de l'instrument du recueil des données. Puis, nous donnerons quelques exemples des activités de conscientisation pratiquées en classe au cours de l'expérimentation. Et pour finir, nous dirons quelques mots sur les résultats obtenus et sur les conclusions auxquelles nous sommes arrivées. 


\section{Problématique de la recherche}

\subsection{La conscientisation grammaticale}

3 Pour la plupart des enseignants, l'apprentissage de la grammaire consiste à développer chez l'apprenant un contrôle automatique de structures grammaticales d'une L2 afin qu'il puisse les employer correctement dans ses productions spontanées. Le développement de ces automatismes langagiers est traditionnellement assuré par la pratique en classe des structures morpho-syntaxiques d'une L2 qui se réalise, tout d'abord, dans des conditions contrôlées (exercices) et puis, dans des contextes de plus en plus proches à la communication naturelle (activités de communication).

4 L'idée de l'efficacité de la pratique se base sur la supposition issue du béhaviorisme que les structures grammaticales s'intériorisent au cours de la répétition renforcée, l'automatisation progressive commençant par la production contrôlée et finissant par la production automatique. Cependant, cette supposition ignore la complexité des processus impliqués dans l'acquisition des connaissances en privilégiant la construction implicite de représentations grammaticales et leur intégration inconsciente dans la grammaire mentale de l'apprenant et en laissant de côté le stade d'aperception et de comparaisons conscientes.

5 L'insuffisance de la pratique pour la structuration et l'intériorisation adéquate de phénomènes grammaticaux d'une L2 a été dévoilée dans un certain nombre d'études en acquisition des langues secondes (Rebuffot, 1993 ; Harley \& Swain, 1984 ; Ellis, 1984 ; Ellis \& Rathbone, 1987) où il a été montré que les activités communicatives pratiquées en classe n'assuraient pas un usage productif des aspects formellement marqués d'une langue étrangère. Les erreurs dues en partie à l'interférence de la langue maternelle et, en partie, à la surgénéralisation et à la simplification des règles d'une langue étudiée se fossilisaient et le niveau de précision grammaticale atteint par la plupart des apprenants restait très bas.

6 Cette situation a fait repenser le rôle de la conscience (ce qui n'est pas nouveau en soi) en apprentissage du système d'une L2 ce qui a abouti à l'apparition des approches favorisant l'introduction dans le cadre communicatif des activités de la réflexion sur la langue étudiée. On peut parler ici des exercices de conceptualisation (Besse \& Porquier, 1991), de grammar awareness activities (Celce-Murcia \& Hilles, 1988), de l'association des structures grammaticales aux tâches communicatives (Mackey, 1994), de consciousness-raising (Ellis, 1991).

7 Parmi cette panoplie de possibilités, pour notre expérimentation nous avons choisi la "conscientisation grammaticale" de R. Ellis (1997 : 160) qui définit les tâches conçues dans le cadre de cette approche comme "les activités pédagogiques au cours desquelles l'apprenant, exposé aux données en L2, doit opérer ces données de sorte à élaborer une connaissance explicite de certaines propriétés linguistiques de la langue cible ${ }^{1 "}$. 
8 Cette approche est orientée vers la formation chez l'apprenant de connaissances explicites, déclaratives - une sorte de connaissance intellectuelle sur un sujet quelconque.

Pour R. Ellis (1991), les caractéristiques essentielles de la conscientisation grammaticale sont les suivantes :

- Les activités tendent à isoler le phénomène grammatical enseigné, afin d'y attirer l'attention de l'apprenant.

- Le phénomène enseigné est illustré par des exemples, l'apprenant a accès à des règles, à des explications.

- L'apprenant doit faire un effort intellectuel pour comprendre le fonctionnement du phénomène étudié.

- Si l'apprenant n'arrive pas à saisir, à comprendre le fonctionnement du phénomène grammatical, l'enseignant prévoit des activités de clarification (c'est-à-dire que l'enseignant modifie, adapte, ajoute de l'information). Parfois on demande aux apprenants de formuler explicitement les règles décrivant le fonctionnement du phénomène étudié.

10 Il faut aussi souligner que la conscientisation grammaticale favorise le recours à la L1 et la "comparaison des langues mises en contact", ce qui est actuellement $\mathrm{vu}$ "comme un instrument efficace, suscitant des verbalisations et des conceptualisations et développant ainsi la conscience linguistique de l'apprenant" (Véronique, 2005).

\subsection{La catégorie de la détermination}

11 Notre choix de la catégorie de la détermination se justifie par la difficulté de l'appropriation de cette catégorie par les apprenants dont la langue est privée de l'article. Ceci est constaté dans un grand nombre d'études en ALS consacrées à ce problème. La citation suivante de DeKeyser reflète nettement le point de vue de plusieurs chercheurs sur cette question :

Articles (...) are notoriously hard to acquire for native speakers of L1 that do not have them or that use a very different system (Jarvis, 2002; Lui \& Gleason, 2002; Robertson, 2000; Tarone \& Parrish, 1988; Thomas, 1989; Young, 1996; cf. also Celce-Mutcia \& Larsen-Freeman, 1999, chap. 15). This element of grammar is strongly resistant to instructional treatments (for articles see, e.g., Butler, 2002 Master, 1997).

Articles express highly abstract notions that are extremely hard to infer, implicitly or explicitly, from the input.

Where the semantic system of the L1 is different from that of L2, as is very often the case for aspect, or where equivalent notions do not expressed overtly in L1, except through discourse patterns, as may be the case for ESL articles for native speakers of most Slavic languages, the learning problem is serious and long-lasting (DeKeyser, 2005: 5).

Les apprenants russophones ne font pas exception vis-à-vis de ce constat. L'absence en russe d'un équivalent morphologique de l'article français, l'asymétrie des moyens d'expression de la catégorie de la détermination (il s'agit de la composante morphologique en français contre la composante 
discursive, syntaxique et lexicale en russe, ce qui forme un dispositif pédagogique disparate), le fait que le russe, à la différence du français, ne tient pas compte des connaissances partagées par le locuteur / auditeur dans la situation de communication et accorde moins d'importance à l'expression de la dichotomie partie / tout, explique le taux relativement bas de l'appropriation de ce phénomène par les apprenants, même dans le contexte des études spécialisées.

\subsection{La conscientisation grammaticale pour l'enseignement de la catégorie de la détermination}

13 L'utilisation de cette approche dans notre contexte d'apprentissage nous paraît justifiée pour plusieurs raisons. Tout d'abord, dans notre cas, il s'agit d'apprenants en première année. Certains d'entre eux sont de vrais débutants. Pour eux, la comparaison explicite du système grammatical de la langue cible avec celui de la langue source constitue, selon nous, un moyen efficace de construire assez rapidement une représentation mentale de ce système.

En plus, nos étudiants possèdent des connaissances métalinguistiques dans leur langue maternelle qui sont exigées au concours d'entrée à l'université, ce qui leur facilite l'aperception des particularités linguistiques dans les deux langues ainsi que la réflexion grammaticale. Ce recours à la comparaison de la langue de départ avec la langue étrangère nous paraît d'autant plus logique qu'il peut s'appuyer sur les stratégies d'apprentissage qui amènent les apprenants à s'interroger sur les convergences et les divergences entre la L1 et la L2. Il faut dire que ces stratégies spécifiques sont développées chez nos apprenants, maîtrisant au moment de l'entrée à l'université au moins une langue étrangère.

Ensuite, il s'agit dans notre cas des apprenants adultes dont le système cognitif atteint son point maximal en ce qui concerne l'attention, la concentration, la mémoire, les aptitudes d'analyse ou de généralisation.

Puis, les interprètes n'ont pas le droit à l'erreur, ce qui nous amène à ne pas nous fier entièrement à l'assimilation inconsciente du système conceptuel étranger.

17 Finalement, soulignons-le encore une fois, le fonctionnement de la catégorie de la détermination est difficile à induire directement des données langagières sans réfléchir et discuter en classe.

\section{La méthodologie de recherche}

\subsection{Les hypothèses de la recherche}

18 L'objet de notre recherche s'inscrit dans le cadre de la problématique formulée par H. Besse : "Dans quelle mesure l'apprentissage d'une description ou simulation grammaticale favorise-t-il ou entrave-t-il l'intériorisation de la grammaire d'une L2 ?" (Besse \& 
Porquier, 1991), ou autrement dit, "dans quelle mesure les connaissances grammaticales influencent-elles la compétence grammaticale" (Cuq \& al., 2005 : 75).

Ainsi dans notre recherche, nous tenons à examiner deux questions générales :

La première est la suivante: est-ce que l'enseignement / apprentissage explicite de l'article par le biais des activités de conscientisation amène à son intériorisation par le public spécialisé dans l'apprentissage des L2 ?

La deuxième consiste à trouver s'il existe des cas d'emploi de l'article qui sont plus difficiles que les autres (qui s'acquièrent moins vite, voire ne s'acquièrent pas).

Dans le présent article, nous n'aborderons que la première question. À notre avis, deux hypothèses peuvent être formulées à ce sujet.

La première hypothèse est que les activités de conscientisation au cours de l'enseignement / apprentissage du public, ayant une base linguistique théorique, peuvent jouer un rôle indirect dans l'intériorisation d'un nouveau phénomène grammatical parce que ces activités favorisent l'aperception et la comparaison des valeurs sémantiques de ce phénomène dans la langue maternelle et la langue étudiée.

La deuxième hypothèse est que les activités de conscientisation comprenant des discussions en classe et suscitant un effort cognitif important auront un effet à long terme.

\subsection{Nos terrains}

5 Pour vérifier nos hypothèses, nous avons prévu l'exploitation de deux terrains. Le premier terrain représente un groupe d'étudiants étrangers apprenant le français en milieu endolingue (Université de Technologie de Compiègne). Ces étudiants nous ont servi de groupe - témoin.

Le deuxième terrain est un groupe d'apprenants russes (université linguistique de Nijni Novgorod) qui a participé à l'apprentissage de l'article au cours des activités de conscientisation.

27 Tout d'abord nous croyons nécessaire de dire quelques mots sur la spécificité des publics que nous comparons dans notre recherche.

En ce qui concerne le terrain russe, ce sont des étudiants monolingues, de 17-20 ans, majoritairement des filles. L'objectif de leur apprentissage consiste à maîtriser tous les aspects de la langue - compréhension (orale et écrite), expression (orale et écrite), lexique, grammaire, culture - c'est-à-dire à développer d'une manière équilibrée toutes les composantes de la compétence communicative. Leur cursus comprend des matières théoriques comme linguistique, phonétique théorique, lexicologie, histoire de la langue, grammaire théorique, stylistique et des matières pratiques comme cours de langue et cours de traduction et d'interprétariat. Le nombre d'heures pour des cours de langue est vraiment considérable; par exemple, pour les étudiants-interprètes qui apprennent le français comme première langue 
étrangère, ce nombre constitue approximativement quatre cents heures par an. Ces étudiants sont traditionnellement orientés vers l'attention à la forme, c'est-à-dire vers l'apprentissage de la structure grammaticale d'une L2 du fait qu'en milieu exolingue, il est plus difficile de travailler sur la compréhension orale et sur le côté lexical, surtout en ce qui concerne la communication courante. Ils sont motivés par leur intérêt pour les langues et cultures étrangères et par le fait que ce sera leur métier.

Pour le terrain français, ce sont des étudiants étrangers, multilingues (essentiellement, des Portugais et des Espagnols, bien qu'il y ait des Anglais, des Chinois, des Coréens, un Persan et une Slovaque), de 18-25 ans, des deux sexes. Ces étudiants sont de futurs ingénieurs, donc leur objectif consiste à développer les compétences de compréhension (orale et écrite) et d'expression (orale et écrite) pour obtenir de nouvelles connaissances dans leurs domaines et mener à bien leurs études en France. Certains d'eux cherchent aussi à prendre connaissance de la culture française et à trouver des amis français. Généralement, ils ne sont pas intéressés à travailler sur la précision grammaticale et la moitié d'entre eux croit que faire des exercices de grammaire n'est pas très utile pour apprendre une L2. Cependant, ils sont unanimes dans la conviction que l'apprentissage de règles de grammaire est nécessaire pour apprendre à parler une langue étrangère.

30 Ainsi, nous pouvons dire que nos terrains sont comparables au niveau psycho-physique - âge, développement cognitif, niveau de scolarisation et au niveau de leurs connaissances en français, mais sont un peu différents en ce qui concerne leurs objectifs d'apprentissage.

\subsection{Instrument du recueil des données}

31 Afin de contrôler l'évolution de l'emploi de l'article par nos terrains, nous avons conçu un pré-test et un post-test semblables se composant de tâches suivantes :

- $1^{\text {ère }}$ tâche : textes à trous (à insérer l'article convenable) ;

- $2^{\text {ème }}$ tâche : jugement de grammaticalité ;

- $3^{\text {ème }}$ tâche : explication de l'emploi / du choix de tel ou tel article ;

- $4^{\text {ème }}$ tâche : un sujet à développer (pour le contexte français - décrire le premier jour du séjour en France ; pour le contexte russe - décrire le jour de la rentrée (en Russie, c'est le 1 septembre).

Entre le pré-test et le post-test, les étudiants en France ont suivi un stage intensif de langue qui a duré 3 semaines ( 6 heures de français par jour, ce qui fait 30 heures de français en tout). Ce stage se concentrait surtout sur la formation des compétences de compréhension et d'expression. La compétence grammaticale (y compris l'emploi de l'article) ne se trouvait pas au centre des préoccupations des enseignants pendant ce stage. Le groupe des étudiants russes a eu 12 heures de cours sur l'emploi de l'article répartis sur un mois. Pendant ce temps-là, les apprenants continuaient à assister à leurs 
cours de français habituels (en général, 12 heures de cours par semaine, ce qui fait 48 heures de français en tout).

\section{Les activités de conscientisation pratiquées en classe}

Sur le plan pratique, les cours en Russie comportaient les éléments suivants : premièrement, la discussion / la mise en question des modèles théoriques traitant le problème de l'article dans la perspective contrastive. Deuxièmement, des activités de comparaison du fonctionnement de la catégorie de la détermination dans la L1 et dans la L2, ainsi que l'analyse de textes et la vérification des hypothèses sur les effets du sens produits par les déterminants en L1 et en L2. Troisièmement, des discussions sur les textes étudiés : sujets, problèmes soulevés, idées évoquées.

\subsection{Modèles théoriques étudiés}

En ce qui concerne la discussion des modèles théoriques, le travail de l'enseignant consistait à les adapter et à les présenter. Les étudiants, de leur côté, étaient censés y découvrir des points communs et des différences et les présenter sous forme de schémas / tableaux. Ils devaient aussi découvrir si les catégories lexico-grammaticales figurant dans ses modèles faisaient partie du système de la L1.

Quels étaient ces modèles? Dans le choix des modèles théoriques à étudier, nous avons donné la préférence aux modèles hypothético-déductifs qui s'interrogent sur la nature et le fonctionnement des opérations mentales sous-jacentes aux usages que l'on fait des formes (y compris l'article), non seulement en français mais dans le plus grand nombre possible des langues. Plus précisément, il s'agit de trois modèles. Le premier est celui de G. Guillaume (1964) où les différentes formes de l'article servent à exprimer deux mouvements de pensée opposés - le mouvement de "particularisation" et le mouvement de "généralisation". Le deuxième est celui de B. Pottier (1974) pour qui la détermination exprime la chronologie des événements, l'opposition nouveau/ connu. Le troisième est le modèle d'A. Culioli (Culioli, Fuchs \& Pêcheux, 1970) pour qui l'ensemble des déterminants représente la trace en surface d'opérations mentales profondes d'extraction, de fléchage et de parcours.

Ces trois modèles nous paraissent intéressants parce que malgré leur appareillage conceptuel différent, l'idée de départ est toujours la même. Ils tentent tous de simuler, abstraitement et hypothétiquement, un mécanisme conceptuel qui permet de rendre compte de certaines des valeurs sémantiques qui peuvent se retrouver dans un grand nombre d'autres langues.

37 Une telle approche crée une base linguistique théorique permettant d'avoir une vision plus générale de la notion de "langue" ainsi que de la catégorie 
sémantique de la détermination, et de commencer l'apprentissage à partir du repérage des sens communs vers ce qui est différent.

De ce point de vue, il serait également intéressant de fusionner le schéma résumant les valeurs de l'article non défini présenté par R. Martin (1992) et, notamment, la partie de ce schéma concernant l'opération de l'extraction par rapport à un ensemble d'objets (en gros caractères ci-dessous) avec la carte sémantique de différents types des indéfinis proposé par M. Haspelmath (1997) pour la langue anglaise (dans notre dessin, les valeurs de l'article indéfini anglais sont en Arial).

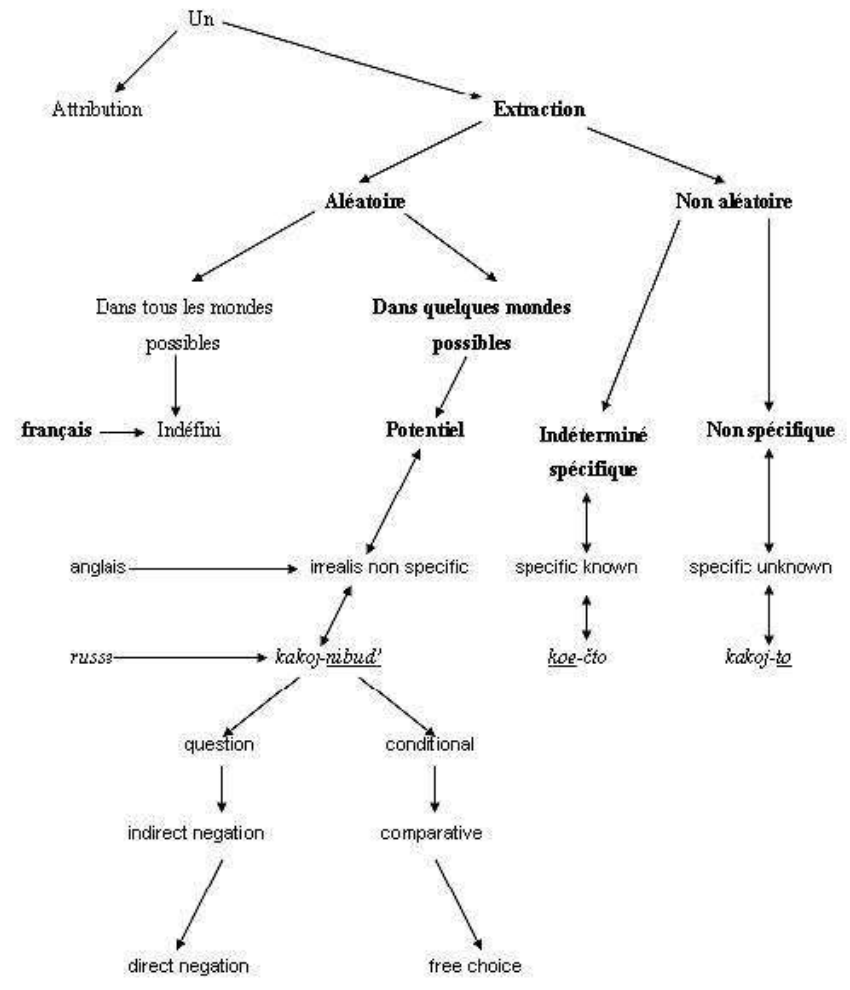

On peut facilement constater que les valeurs sémantiques exprimées par l'article indéfini en français sont les mêmes que dans la langue anglaise ("potentiel" exprimant la même chose que "irrealist non specific", "indéterminé spécifique" étant égal à "specific known" et "non spécifique" représentant l'équivalent de "specific unknown"). Il faut aussi souligner qu'en français aussi bien qu'en anglais, ces différentes valeurs sont exprimées par la même forme de l'article: $\boldsymbol{u}$ en français et $\boldsymbol{a}$ en anglais. La même phrase proposée par R. Martin, Mon ami veut épouser une Portugaise, peut illustrer ses trois valeurs différentes pour le français et pour l'anglais :

- Mon ami veut épouser une Portugaise/ My friend wants to marry with a Portuguese (n'importe laquelle, existant sous une forme conceptuelle plutôt que réelle)

- Mon ami veut épouser une Portugaise/ My friend wants to marry with a Portuguese (que moi, le locuteur, je connais)

- Mon ami veut épouser une Portugaise/ My friend wants to marry with a Portuguese (que moi, le locuteur, je ne connais pas, mais cette Portugaise est une fille concrète, réelle). 
Mais ce qui est encore plus intéressant pour nous, c'est que d'après certaines recherches en linguistique contrastive (Seliverstova, 1964; Padučeva, 1974, 1985 ; Partee, 2005), la catégorie de la détermination fait partie des universaux linguistiques et certains des concepts de cette catégorie, représentés en français par l'article indéfini, reçoivent les formes de pronoms indéfinis en russe (les séries régulières en russe sont en italique). Ces valeurs exprimées en français et en anglais par un article indéfini reçoivent trois formes différentes en russe: séries de pronoms en -nibud' pour "potentiel", séries de pronoms en -koe pour "indéterminé spécifique" et séries de pronoms en -to pour "non spécifique". Ainsi, le russe apparaît comme une langue qui au niveau des formes présente plus de régularité pour la relation : forme - sens. Cependant, il faut noter qu'en russe, il n'est pas obligatoire de se servir de pronoms indéfinis pour exprimer ces sens-là. La même phrase - Mon ami veut épouser une Portugaise - pourrait être traduite en russe sans pronom indéfini. Ainsi l'absence de pronom indéfini devant un nom permet d'avoir les trois lectures différentes citées antérieurement - "potentiel", "indéterminé spécifique" et "non spécifique", tandis que l'emploi d'un des pronoms indéfinis (kakoj-nibud', koe-kakoj, kakoj-to) rend inutile la référence au contexte.

Ainsi l'enseignement / apprentissage de la catégorie de la détermination peut être amorcé par la comparaison des effets du sens exprimés par l'article français avec le système des pronoms indéfinis dans la langue russe, ce qui permettrait à notre avis de développer chez les apprenants russophones une certaine idée du fonctionnement de l'article indéfini français se fondant sur leur intuition de natif, ce qui facilitera la conceptualisation de la catégorie de la détermination et aboutira à la formation d'un "système des effets sémantiques" à visée universaliste.

En ce qui concerne les cas d'emploi de l'article n'ayant pas d'équivalents dans la langue maternelle des apprenants, ils pourraient être discutés ultérieurement.

\subsection{Exemples des exercices de conscientisation}

Voilà quelques exemples de consignes d'exercices de conscientisation que nous avons proposés à nos étudiants afin de les sensibiliser à l'universalité des concepts sémantiques exprimés par l'article :

- Indiquer les phrases correctes / incorrectes en langue maternelle (russe), expliquer quels éléments rendent ces phrases incorrectes (Exemple: Pjat'jablok polezny dlja zdorovija = Cinq pommes sont utiles pour la santé).

- Indiquer si les pronoms indéfinis mis devant les groupes nominaux altèrent le sens de la phrase (en russe), expliquer pourquoi (Exemple: On kupil (kakuju-nibud'/ kakuju-to/ koe-kakuju) = Il a acheté une voiture).

- Grouper les phrases suivantes selon le sens qu'elles expriment (les noms des groupes sont indiqués) - toute la classe des objets; une partie des objets; un objet concret, réel; un objet imaginaire; un objet unique dans la situation donnée; un des objets dans la situation donnée; etc.

Recherches en didactique des langues et des cultures, 6-1 | 2009 
- Analyser les phrases suivantes, dire quels modes, quels temps et quels types de la phrase conviennent aux groupes suivants : la classe des objets, un objet concret, réel; un objet imaginaire; un objet unique dans la situation donnée; etc.

- Souligner les phrases incorrectes, expliquer pourquoi elles paraissent incorrectes en précisant à quel groupe sémantique elles appartiennent (Exemple: Beaucoup de neige est blanche. La neige est blanche. Quelques garçons sont plus courageux que quelques filles. Mon cousin vient d'acheter la nouvelle voiture).

- Remplacer les substituts de l'article dans les phrases correctes et incorrectes par les articles convenables. Associer le sens que l'article peut exprimer avec ses différentes formes.

- Lire le texte, remplacer (où c'est possible) les articles par les mots qui expriment le même sens. Vous pouvez utiliser la liste ci-dessous : ce, peu, une sorte de, mon, certain, quelques, trois, un kilo, différents...

\subsection{Discussion des textes étudiés} expérimental et le groupe - témoin. Les résultats sont les suivants :

\begin{tabular}{|l|l|l|l|l|}
\hline & \multicolumn{2}{|c|}{$\begin{array}{c}\text { Groupe expérimental } \\
\text { (Russie) }\end{array}$} & \multicolumn{2}{c|}{$\begin{array}{c}\text { Groupe témoin } \\
\text { (France) }\end{array}$} \\
\hline Réponses & Correctes & Incorrectes & Correctes & Incorrectes \\
\hline Pré-test (93 cas) & 70 & 23 & 59 & 34 \\
\hline
\end{tabular}




\begin{tabular}{|l|l|l|l|l|}
\hline Post-test $(94$ cas $)$ & 79 & 15 & 63 & 31 \\
\hline Test $(2$ mois plus tard ; 93 cas $)$ & 80 & 13 & --- & --- \\
\hline
\end{tabular}
l'apprentissage de l'article, nous pouvons dire que cette dernière a ses limites et ses avantages. Ses limites sont plutôt liées à son caractère savant, théorique et conscient. L'appareillage conceptuel employé dans les modèles linguistiques est abstrait et les apprenants sont obligés de faire un effort important pour se l'approprier, ce qui peut provoquer une perte de temps et une perte de motivation de la part de certains d'entre eux. Ainsi, le passage du savoir savant au savoir enseigné devient un vrai problème pédagogique. Le recours à la conscience, à la réflexion prive aussi les cours de langue de leur caractère ludique, décontracté. Ils se modifient en une sorte de gymnastique intellectuelle. Il découle que ce type d'apprentissage de la grammaire sollicite un public adulte, initié à la réflexion théorique et ayant un bagage de connaissances linguistiques. La deuxième limite consiste dans le fait que la conscientisation grammaticale ne vise pas l'emploi correct des phénomènes grammaticaux appris dans la communication courante, ce qui dévalorise un peu cette approche aux yeux des enseignants et des apprenants.

51 Cependant, les avantages présentés par cette approche nous paraissent plus importants que ses inconvénients. Tout d'abord, elle favorise l'aperception et la comparaison qui forment la base de l'intégration du nouveau phénomène grammatical (mentionnons tout de même que, pour que l'intégration ait lieu, il faut que l'apprenant soit cognitivement prêt).

52 Puis, si l'apprenant est encore incapable d'intégrer de nouvelles connaissances, il peut se construire une représentation explicite plus ou moins structurée qu'il laissera à part, en attendant qu'il soit prêt à l'intégrer. Cette connaissance explicite l'aidera à s'apercevoir du phénomène dans les données langagières auxquelles il sera exposé, ce qui peut faciliter son intégration ultérieure. aguets contre les erreurs possibles et empêche également la fossilisation.

4 Sur le plan pratique, ce type de connaissance peut aider l'apprenant à faire un test de grammaire ou à préparer une intervention, ce qui n'est pas 
négligeable dans les situations où il s'agit d'études dans des établissements d'enseignement supérieur à l'étranger ou de travail dans des entreprises étrangères.

Sur le plan du développement cognitif, les activités de conscientisation grammaticale permettent aux étudiants d'apprendre à généraliser et à systématiser ce qui pourra leur être utile dans le traitement de l'information et dans l'appropriation de connaissances sur tous les sujets.

\section{Conclusion}

Suite à l'analyse de nos données, nous ne pouvons pas attribuer l'amélioration de l'emploi de l'article par le groupe expérimental exclusivement à nos activités pédagogiques. Les facteurs qui influent sur le processus d'apprentissage sont trop nombreux (ce sont, par exemple, différentes variables individuelles ou l'accès à la langue). Nous ne pouvons pas contrôler, mesurer et délimiter l'effet de ces facteurs pour dire quel était l'apport pur de la méthode appliquée. Mais en même temps, nous ne pouvons pas non plus affirmer que cette méthode n'a pas eu d'impact sur nos apprenants, parce que les résultats obtenus font voir qu'il y a eu une amélioration immédiate qui s'est maintenue, même après deux mois d'arrêt de cours. Ainsi, pourrions-nous soumettre nos activités à titre d'expérience pour les enseignants travaillant avec des publics spécialisés comme le nôtre.

\section{BIBLIOGRAPHIE}

Bailly, D. (1998). "Didactique scolaire : le rôle de la conceptualisation grammaticale dans l'apprentissage de la compréhension et de l'expression en langue étrangère". ELA, ํo 111. pp. 325-342.

Besse, H. \& Porquier, R. (1991). Grammaires et didactique des langues. Paris : Didier.

Coste D. (2001). "De plus d'une langue à d'autres encore, penser les compétences plurilingues". In Castellotti, V. (dir.). D'une langue à d'autres : pratiques et représentations. Rouen : Publications de l'Université de Rouen.

Celce-Murcia M. \& Hilles, S. (1988). Techniques and resources in teaching grammar. Oxford: Oxford University Press.

Culioli, A., Fuchs, C. \& Pêcheux, M. (1970). Considérations théoriques à propos du traitement formel du langage. Tentative d'application au problème des déterminants. Paris : Dunod.

Cuq, J.-P. \& Queffelec, A. (2005). "Enseignement de la grammaire et enseignement grammaticalisé en français langue seconde". Le français dans le monde. pp. 75-84.

Cyr P. \& Germain, C. (1998). Les stratégies d'apprentissage. Paris : Clé international. 
DeKeyser, R. (2005). "What Makes Learning Second-Language Grammar Difficult? A Review of Issues". Language Learning, vol. 55, supplément 1. pp. 1-25.

Ellis, R. (1984). " Can syntax be taught? A study of effects of formal instruction on the acquisition of Wh questions by children". Applied Linguistics, vol. 5, no 2 . pp. 138-155.

Ellis, R. \& Rathbone, M (1987) The acquisition of German in a classroom context. Mimeograph. London: Ealing College of Higher Education.

Ellis, R. (1991). "Grammar Teaching - practice or Consciousness-Raising?". In Second Language Acquisition \& Language Pedagogy. Clevedon-Philadelphia-Adelaide: Multilingual Matters LTD.

Ellis, R. (1997). SLA Research and Language Teaching. Oxford: Oxford University Press.

Germain, C. \& Séguin, H. (1998). Le point sur la grammaire. Paris : Clé international.

Guillaume, G. (1964). Langage et science du langage. Paris, Québec, Nizet : Presses de l'Université Laval.

Harley, B. \& Swain, M. (1984). "The interlanguage of immersion and its implications for second language teaching". In Davies, A., Criper, C. \& Howatt, A.P.R. (dir.). Interlanguage Edinburgh: Edinburgh University Press. pp. 291-311.

Haspelmath, M. (1997). Indefinite Pronouns. Oxford: Oxford University Press.

Mackey, A. (1994). "Targeting morpho-syntax in children's ESL: An empirical study of use of interactive goal-based tasks". Working Papers in Educational Linguistics, 10 (1). pp. 67-91.

Martin, R. (1992). Pour une logique du sens. Paris: PUF.

Nitto, R. \& Gardner, S. (2005). "Consciousness-raising and practice in ELT course-books". English Language Teaching Journal, vol. 59, nํ․ 1. pp. 3-13.

Padučeva, E. V. (1974). O semantike sintaksisa: materialy k transformacionnoj grammatike russkogo jazyka [On the Semantics of Syntax: Materials towards the Transformational Grammar of Russian]. Moscou: Nauka.

Padučeva, E. V. (1985). Vyskazyvanie i ego sootnesennost's dejstvitel'nost'ju [The Utterance and its Correspondence with Reality]. Moscou: Nauka.

Partee, B. (2005). "Semantic Typology of Indefinities II". Lecture 6, MGU.

Pottier, B. (1974). Linguistique générale. Théorie et pratique, Paris : Klincksieck.

Rebuffot, J. (1993). Le point sur l'immersion. Anjou, Québec : Centre Educatif et Culture.

Santagcroce M. (2000). Vers une grammaire transitionnelle : faire de la grammaire ou laisser la grammaire se faire? ELA, no 120 . pp. 433-438.

Seliverstova, O. N. (1964). "Opyt semantičeskogo analiza slov tipa vse i tipa kto-nibud' ". [Towards a semantic analysis of words of the vse-type and on the kto-nibud'-type] Voprosy jazykoznanija, no 13. pp. 80-90.

Véronique, D. (2005). "Comparer les langues : perspectives didactiques ?". Le français dans le monde .pp. 18-25.

Viselthier, B. (2001). "Expérimentation de conceptualisation grammaticale dans l'enseignement de l'allemand en France". ELA, no 122. pp. 197-210. 


\section{NOTES}

1. Ellis defines grammar consciousness-raising tasks as "a pedagogic activity where the learners are provided with L2 data in some form and required to perform some operation on or with it, the purpose of which is to arrive at an explicit understanding of some linguistic properties of the target language" (Ellis, $1997: 60)$.

\section{RÉSUMÉS}

Notre recherche porte sur l'utilisation en classe de langue des activités de la conscientisation grammaticale visant l'intériorisation de l'article, phénomène grammatical réputé difficile, voire impossible à s'approprier pour les adultes. Notre public représente un groupe de futurs interprètes, spécialistes de langues. Parmi les activités de conscientisation pratiquées nous avons surtout privilégié celles qui favorisent la comparaison explicite du fonctionnement de la catégorie de la détermination en français et en russe au niveau de modèles théorique et discursif. Les résultats obtenus au cours de l'expérimentation permettent de constater que cette approche peut être efficace pour le public spécialisé en ce qui concerne l'amélioration de la précision grammaticale et le maintien des acquis dans l'expression écrite, bien que son application se heurte à certaines difficultés d'ordre cognitif.

Despite high frequency and early input, French articles remain a generally acknowledged marked feature for [-Article] Russian learners. This paper reports on acquisition of this feature by future interpreters through consciousness raising tasks, focused on calling learners' attention to comparison of discourse patterns between Russian and French in order to construct an explicit understanding of the form-function mapping of articles. The results of the study showed that this approach may be considered a facilitator for the acquisition of articles for specialised learners, in terms of accuracy and long-term effect in prepared speech, though the application of the approach can give rise to some cognitive difficulties.

\section{INDEX}

Mots-clés : apprentissage de la grammaire, activités de conscientisation grammaticale, comparaison, catégorie de la détermination, apprenants russophones, linguistique contrastive, modèles linguistiques théorique, modèles discursifs

Keywords : grammar teaching, consciousness-raising tasks, comparison, articles, Russian learners, contrastive linguistics, theoretical linguistic models, discourse patterns 


\section{AUTEUR}

\section{OLGA SPIRIDONOVA}

Olga Spiridonova est doctorante en didactique des langues étrangères au laboratoire COSTECH de l'Université de Technologie de Compiègne.

Courriel : olgaspir[at]yahoo.fr.

Adresse : Laboratoire LIDILEM, UFR des Sciences du Langage, Université Stendhal Grenoble III, BP 25 - 38040 Grenoble cedex 9. 